\title{
Os benefícios da Odontologia Hospitalar para a população: Uma revisão de escopo
}

\author{
The benefits of Hospital Dentistry for the population: A scope review \\ Los beneficios de la Odontología Hospitalaria para la población: Una revisión de alcance
}

Recebido: 21/03/2021 | Revisado: 31/03/2021 | Aceito: 02/04/2021 | Publicado: 12/04/2021

\author{
Sylvia Cunha Rocha \\ ORCID: https://orcid.org/0000-0002-2023-538X \\ Universidade Federal de Minas Gerais, Brasil \\ E-mail: sylviarocha6@gmail.com \\ Denise Vieira Travassos \\ ORCID: https://orcid.org/0000-0003-2084-9557 \\ Universidade Federal de Minas Gerais, Brasil \\ E-mail: dtravassos@ufmg.br \\ Najara Barbosa da Rocha \\ ORCID: https://orcid.org/0000-0003-3366-0032 \\ Universidade Federal de Minas Gerais, Brasil \\ E-mail: najaraufmg@ufmg.br
}

\begin{abstract}
Resumo
Introdução: Não há clareza de todos os benefícios resultantes da atenção odontológica no ambiente hospitalar. Objetivo: Mapear as evidências dos benefícios de intervenções do cirurgião-dentista em ambiente hospitalar para a população. Metodologia: Revisão de escopo de literatura, utilizando as bases Medline, Biblioteca Virtual de Saúde e Google Scholar, com os descritores: Atenção Odontológica; Saúde Bucal; Equipe Hospitalar de Odontologia; Unidade Hospitalar de Odontologia; Hospital. Critérios de inclusão: intervenções realizadas pelo cirurgião-dentista em ambiente hospitalar; sem restrição de período. Idiomas: português, inglês e espanhol. Endnote Web foi utilizado para manipulação de referências. Resultados: A busca identificou 4349 resumos de artigos, sendo 111 selecionados na triagem. Foram lidos 48 textos, resultando 11 estudos. Os estudos foram realizados com pacientes de diversas faixas etárias e/ou com diferentes doenças e condições. Os benefícios foram: prevenção de pneumonia, focos infecciosos, osteorradionecrose, infarto do miocárdio; redução de mucosite oral no tratamento de câncer, com melhoria da qualidade de vida; diminuição da internação e custo hospitalar. Conclusão: São valiosos os benefícios que o odontólogo traz para população no ambiente hospitalar e para o estabelecimento de saúde, sendo que sua presença neste local deve ser estimulada.
\end{abstract}

Palavras-chave: Atenção odontológica; Saúde bucal; Equipe hospitalar de odontologia; Unidade hospitalar de odontologia; Hospital.

\begin{abstract}
Introduction: It is not clear all the benefits resulting from dental care in hospital environment. Objective: To map the evidence of the benefits of dentistry interventions in hospital settings for the population. Metodology: Literature scope review, using the databases Medline, Biblioteca Virtual de Saúde and Google Scholar, with the descriptors: Dental Care; Oral Health; Dental Staff; Hospital Dentistry Unit; Hospital. Inclusion criteria: interventions performed by the dental surgeon in a hospital environment; no period restriction. Languages: Portuguese, English and Spanish. Endnote Web was used for reference manipulation. Results: The search identified 4349 abstracts of articles, and 111 were selected in the screening. Forty-eight texts were read, resulting in 11 studies. The studies were conducted with patients of various age groups and/or with different diseases and conditions. The benefits were: prevention of pneumonia, infectious foci, osteoradionecrosis, myocardial infarction; reduction of oral mucositis in cancer treatment, with improved quality of life; reduced hospitalization and hospital costs. Conclusion: The benefits that the dentist brings to the population in the hospital environment and to the health establishment are valuable, and their presence in this place should be stimulated.
\end{abstract}

Keywords: Dental care; Oral health; Dental staff, Hospital; Dentistry hospital unit; Hospital.

\section{Resumen}

Introducción: No están claros todos los beneficios que se derivan de la atención odontológica en el ámbito hospitalario. Objetivo: Mapear las evidencias de los beneficios de las intervenciones del cirujano-dentista en el ambiente hospitalario para la población. Materiales y Metodología: Revisión de la literatura, utilizando las bases Medline, Biblioteca Virtual de Salud y Google Scholar, con los escritores: Atenção Odontológica; Saúde Bucal; Equipe Hospitalar de Odontologia; Unidade Hospitalar de Odontologia; Hospital. Criterios de inclusión: intervenciones realizadas por el cirujano dental en un entorno hospitalario; sin restricción de periodo. Idiomas: portugués, inglés y español. Se utilizó Endnote Web para manipular las referencias. Resultados: La búsqueda 
identificó 4349 resúmenes de artículos, siendo 111 los seleccionados en el cribado. Se leyeron 48 textos, lo que dio lugar a 11 estudios. Los estudios se realizaron con pacientes de diversos grupos de edad y/o con diferentes enfermedades y afecciones. Los beneficios fueron: prevención de neumonía, focos infecciosos, osteorradionecrosis, infarto de miocardio; reducción de la mucositis oral en el tratamiento del cáncer, con mejora de la calidad de vida; reducción de la hospitalización y de los costes hospitalarios. Conclusión: Los beneficios que el odontólogo aporta a la población en el ámbito hospitalario y al establecimiento de salud son valiosos, y su presencia en este lugar debe ser estimulada.

Palabras clave: Salud bucal, Atención odontológica, Personal de odontología den hospital, Servicio odontológico hospitalario, Hospital.

\section{Introdução}

A Odontologia hospitalar é definida como a área de atividade do Cirurgião Dentista em ambiente hospitalar, promovendo atenção odontológica de baixa, média ou alta complexidade a pacientes hospitalizados, objetivando contribuir com a cura e melhora geral da qualidade de vida do paciente (Gaetti-Jardim et al., 2013).

O desenvolvimento da Odontologia hospitalar na América vem ocorrendo desde a metade do século XIX. No Brasil, em 2004, a Odontologia Hospitalar foi legitimada com a criação da Associação Brasileira de Odontologia Hospitalar (AranegaI et al., 2012). Foi reconhecida pelo Conselho Federal de Odontologia, como campo de atuação, no ano de 2015, com a criação da habilitação em Odontologia Hospitalar (Webster, 2016).

Na literatura é apontado por que a odontologia hospitalar no Brasil ainda está se consolidando, com número insuficiente de cirurgiões-dentistas atuando em hospitais (Blum et al., 2018). Já em outros países, como nos EUA, odontológos começaram a trabalhar em ambiente hospitalar desde o início do século XX. Os serviços odontológicos começaram a ser realizados em alguns hospitais estadunidenses, e hoje em dia grande parte desses hospitais possuem dentistas atuando em suas equipes de trabalho (Willis, 1965).

Estudos mostram que a melhoria da higienização bucal reduz significantemente a progressão da ocorrência de doenças respiratórias em pacientes internados em Unidade de Terapia Intensiva (UTI) (Nawata et al., 2019; Rabelo et al., 2010; Sato et al., 2016; Sona et al., 2009), além de reduzir a utilização de antibióticos e consequente mortalidade, resultando em uma queda nos custos dos serviços e diminuição do período de internação (Bellissimo-Rodrigues et al., 2018; DeRiso et al., 1996; Sona et al., 2009).

Não há clareza de todas as vantagens resultantes da atenção odontológica no ambiente hospitalar em um único estudo, com necessidade de evidenciar os reais benefícios da odontologia hospitalar para a população. Assim, este estudo objetivou mapear as evidências disponíveis de intervenções pelo cirurgião-dentista dentro do ambiente hospitalar que trouxeram benefícios para a saúde do paciente.

\section{Metodologia}

\section{Desenho do estudo}

Trata-se de uma revisão de escopo realizada para mapear a literatura sobre os benefícios da odontologia hospitalar para a população. Este tipo de estudo objetiva descrever os resultados dos estudos revisados, sem avaliá- los criticamente (Arksey \& O'Malley, 2005).

Esta revisão de escopo seguiu recomendações propostas pelo PRISMA (Tricco et al., 2018). A revisão envolveu a formulação de uma pergunta de pesquisa para orientar o estudo: "Quais os benefícios da intervenção odontológica em ambiente hospitalar para a população?”. A revisão foi realizada entre em setembro de 2020. 


\section{Estratégias de buscas}

Assim, foram analisados estudos sobre pacientes em ambiente hospitalar (participantes), com intervenções odontológicas realizadas pelo cirurgião-dentista que impacta na saúde dos pacientes (conceito) e em ambiente hospitalar (contexto).

As bases de dados utilizadas foram: Pubmed e Biblioteca Virtual de Saúde, que são bases abrangentes, confiáveis e por incluir estudos internacionais, e Google Scholar que analisou a literatura cinzenta. Os descritores utilizados foram: Atenção odontológica; saúde bucal; higiene oral; equipe hospitalar odontológica; serviço odontológico hospitalar; hospital, utilizando operadores boleanos and e or, de acordo com a Figura 1.

Figura 1. Estratégia de buscas nas bases de dados, 2020.

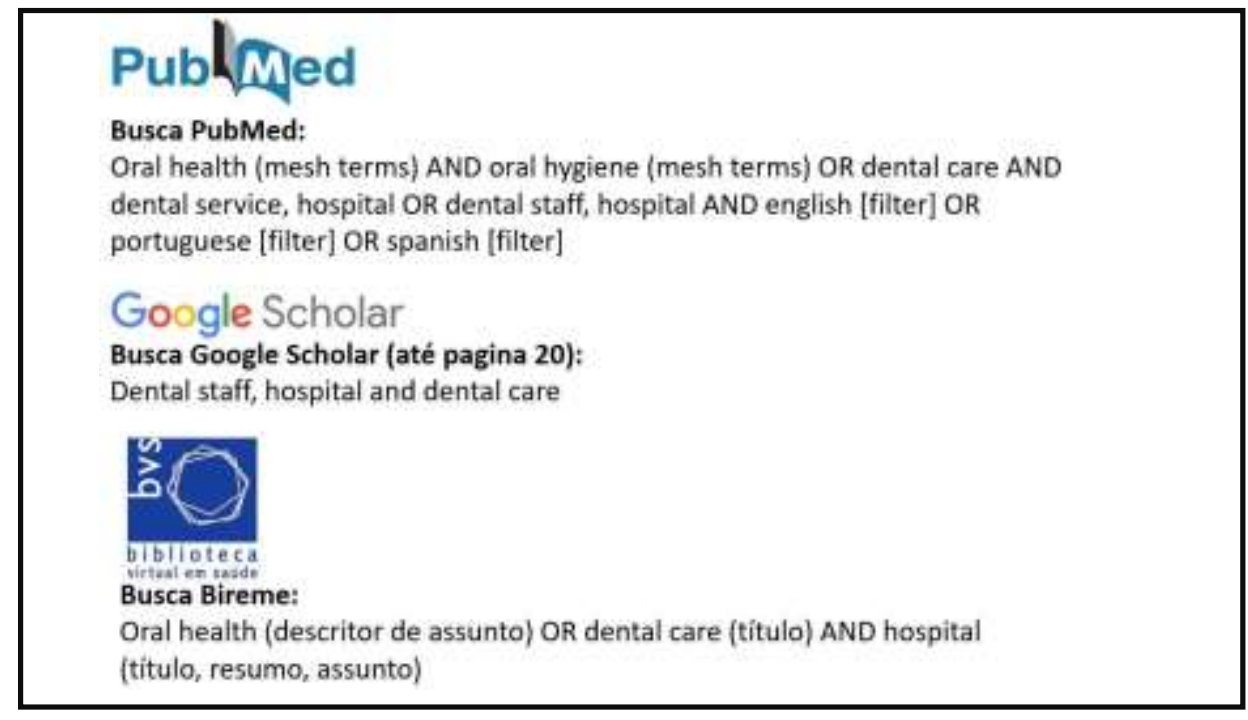

Fonte: Autores.

\section{Critérios de inclusão e exclusão}

Os critérios de inclusão dos estudos foram: ocorridos em ambiente hospitalar e com intervenções realizadas pelo cirurgião-dentista; sem restrição de período, todos os tipos, exceto revisões de literatura; idioma: português, inglês e espanhol. Foram considerados critérios de exclusão: estudos que não citavam os benefícios da Odontologia Hospitalar; estudos que não eram com pacientes hospitalizados; realizados em animais; sem resultados ou não publicados e sem texto completo.

\section{Formação do banco de dados para a revisão de escopo}

Foi criado um banco de dados, com os estudos selecionados na busca de dados, de acordo com: citação completa do artigo, país onde foi realizado o estudo, título, nome da revista, idioma de publicação e resumo. Foi realizada a eliminação das duplicidades, antes do banco ser analisado pelas pesquisadoras.

O programa Endnote foi responsável pela manipulação das referências.

\section{Seleção dos estudos}

A seleção dos estudos foi realizada por dois revisores (SCR, NBR) em duas etapas, sendo a primeira com a seleção dos artigos pelos títulos e em momento posterior, os resumos. Segunda, os textos completos dos artigos selecionados foram obtidos e identificados pela leitura aqueles que respondiam aos objetivos do estudo. As etapas foram realizadas de forma independente pelos dois pesquisadores e, em momento posterior obtido o consenso. 


\section{Análise dos dados}

Os artigos incluídos no estudo tiveram seus textos completos lidos e as seguintes informações foram transcritas para uma planilha em formato Microsoft Excel: nome do artigo, autor, ano de publicação e local, metodologia, principais achados e considerações finais relevantes.

Não houve necessidade de apreciação por Comitê de Ética em Pesquisa devido à natureza do estudo.

\section{Resultados}

\section{Busca}

A busca inicial identificou 4349 resumos de artigos e após a remoção dos resumos duplicados ( $\mathrm{n}=133$ ), foram selecionados 111 artigos na triagem. Com os critérios de elegibilidade, foram incluídos 48 textos completos e após sua leitura, resultaram 11 estudos, que foram inseridos na revisão (Figura 2).

Figura 2. Seleção dos estudos nas bases de dados, 2020.

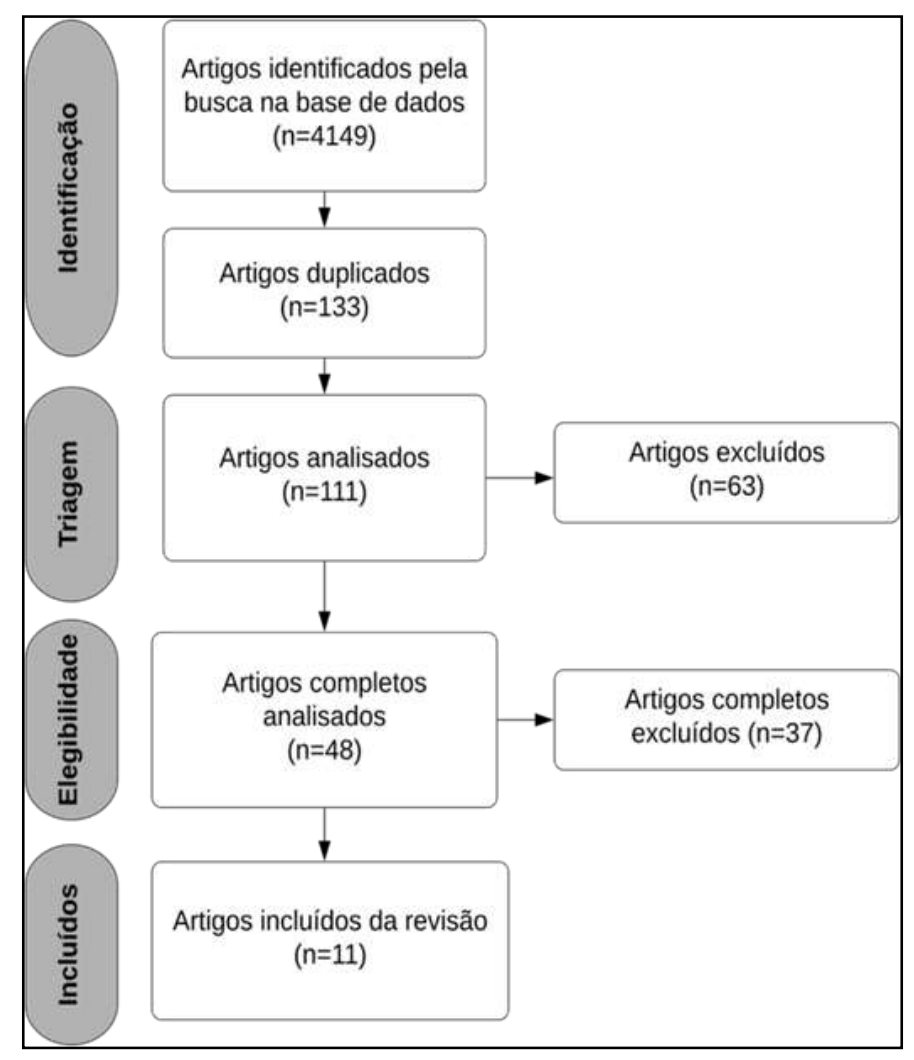

Fonte: Autores.

\section{Caracterização dos artigos científicos}

Dos estudos identificados nesta revisão, de acordo com os critérios de elegibilidade, 5 eram nacionais e 6 internacionais, sendo realizados na Inglaterra, França e Suécia e três estudos no Japão.

Foram encontrados estudos de diferentes metodologias, tais como relatos de casos(Macedo et al., 2019; Miranda \& Montenegro, 2010; Nawata et al., 2019), estudos observacionais transversais (Austriaco-Leite et al., 2018; Frascino et al., 2018; Hashem et al., 2020; Raskin et al., 2018), observacionais longitudinais (Friedlander et al., 2018; Sato et al., 2016; Yokota et al., 2016) e um estudo experimental clínico randomizado (Bellissimo- Rodrigues et al., 2018). 


\section{Principais achados}

Os estudos que mostraram benefícios da odontologia hospitalar foram realizados com pacientes de diversas faixas etárias (crianças até idosos) e/ou com diferentes doenças e condições.

Um dos benefícios da presença do profissional da Odontologia no ambiente hospitalar foi a prevenção de pneumonia ou infecções do trato respiratório (Nawata et al., 2019; Sato et al., 2016), porém outros benefícios foram também evidenciados, tais como: prevenção de endocardite ou focos infecciosos em pacientes pediátricos, cardiopatas, com câncer e/ou em adultos (Bellissimo-Rodrigues et al., 2018; Frascino et al., 2018; Yokota et al., 2016); prevenção de osteorradionecrose (Macedo et al., 2019; Raskin et al., 2018; Yokota et al., 2016) e redução da incidência ou encurtamento da duração de mucosite oral (MO) em pacientes em tratamento de câncer (Frascino et al., 2018; Macedo et al., 2019), com prevenção de efeitos colaterais da terapia antineoplásica e melhoria em sua qualidade de vida (Frascino et al., 2018; Hashem et al., 2020; Macedo et al., 2019; Miranda \& Montenegro, 2010; Nawata et al., 2019; Yokota et al., 2016).

Foi apontado que o cirurgião-dentista pode detectar calcificações placas da artéria carótida por meio do exame radiográfico com a panorâmica e prevenir assim, o infarto do miocárdio (Friedlander et al., 2018). Também foi mostrado que devido ao elevado custo do tratamento de infecções hospitalares, a presença do cirurgião-dentista no hospital resulta em um ótimo custo-benefício para o serviço hospitalar (Bellissimo-Rodrigues et al., 2018).

Os benefícios das intervenções odontológicas encontradas pela revisão de escopo estão na Figura 3, bem como os artigos que as descreveram.

Figura 3. Mapa conceitual sobre os artigos selecionados na revisão de escopo e os benefícios apontados, 2020.

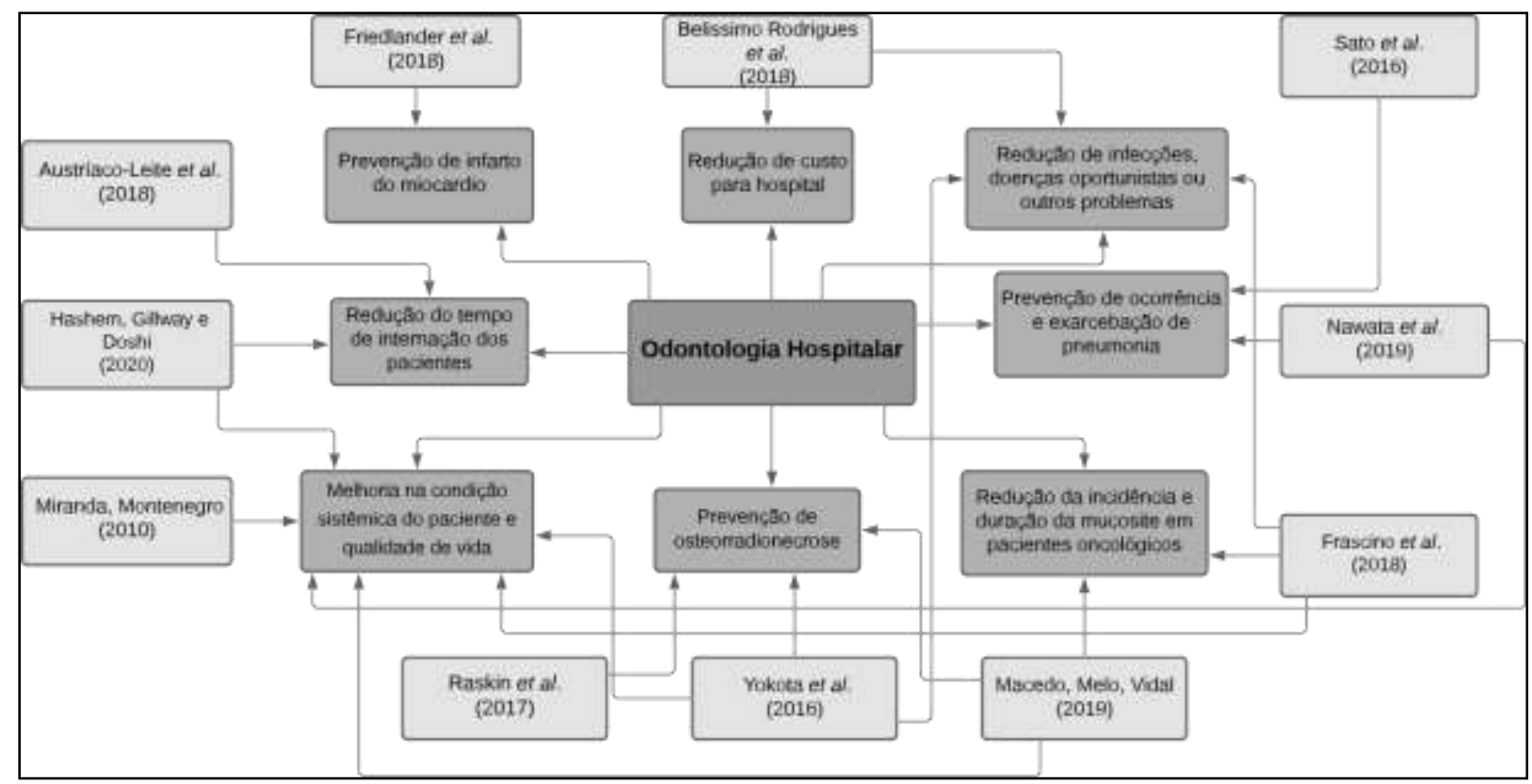

Fonte: Autores.

A Tabela 1 descreve os artigos selecionados na revisão de escopo em relação as suas características. 
Research, Society and Development, v. 10, n. 4, e33410414117, 2021

(CC BY 4.0) | ISSN 2525-3409 | DOI: http://dx.doi.org/10.33448/rsd-v10i4.14117

Tabela 1. Distribuição dos estudos selecionados, de acordo com suas características, 2020.

\begin{tabular}{|c|c|c|c|c|}
\hline Título, Autor e Ano & Tipo de estudo & Metodologia & Principais resultados & Considerações relevantes \\
\hline $\begin{array}{l}\text { Ação odontológica preventiva } \\
\text { em paciente idoso dependente } \\
\text { na Unidade de Terapia } \\
\text { Intensiva (UTI) - Relato de } \\
\text { caso (Miranda \& Montenegro, } \\
\text { 2010) }\end{array}$ & Relato de Caso & 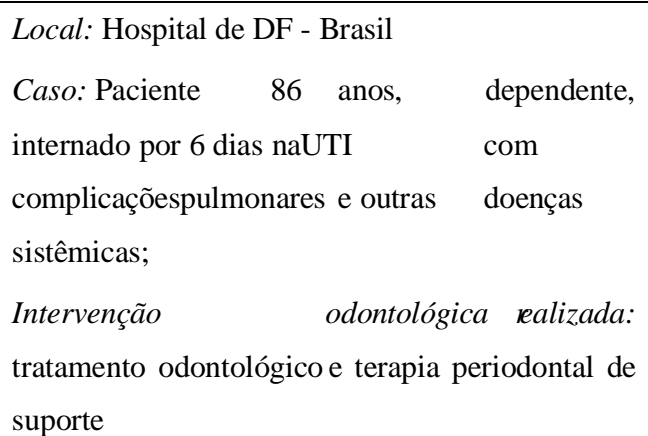 & $\begin{array}{l}\text { Melhora significativa da condição odontológica do } \\
\text { paciente, prevenindo o aparecimento de novas } \\
\text { doenças oportunistas }\end{array}$ & $\begin{array}{l}\text { A participação de um CD capacitado na UTI } \\
\text { melhora quadro clínico paciente prejudiciais à } \\
\text { saúde sistêmica, compondo assim equipes } \\
\text { verdadeiramente interdisciplinares; } \\
\text { Houve uma melhora significativa da condição } \\
\text { odontológica do paciente na UTI, colaborando } \\
\text { para evitar aparecimento dedoenças oportunistas }\end{array}$ \\
\hline $\begin{array}{l}\text { Esophageal cancer patients } \\
\text { have a high incidence of } \\
\text { severe periodontitis and } \\
\text { preoperative dental care } \\
\text { reduces the likelihood of } \\
\text { severe pneumonia after } \\
\text { esophagectomy } \\
\text { (Sato et al., 2016) }\end{array}$ & $\begin{array}{l}\text { Caso controle } \\
\text { observacional }\end{array}$ & $\begin{array}{l}\text { Local: Hospital UniversitárioAkita-Japão; } \\
\text { Período: } 2003 \text { e 2014; } \\
\text { Amostra e intervenção: } 529 \text { pacientes com } \\
\text { cancro esofágico em relação à incidência de } \\
\text { pneumonia grave após esofagectomia - grupo } \\
\text { experimental (232) com tratamentos odontológicos } \\
\text { e grupo controle (297) }\end{array}$ & $\begin{array}{l}\text { Análise dos dados revelou que uma complicação } \\
\text { da cirurgia } \\
\text { gastrointestinal (fuga anastomótica), cuidados } \\
\text { dentários pré-operatórios e sexo estavam } \\
\text { significativamente correlacionados com a } \\
\text { ocorrência de pneumonia grave pós-operatória }\end{array}$ & $\begin{array}{l}\text { Os dentistas devem avaliar e realizaratendimento } \\
\text { odontológico nos pacientes para redução da } \\
\text { probabilidade de pneumonia grave após a } \\
\text { esofagectomia }\end{array}$ \\
\hline $\begin{array}{l}\text { Hospital and oncological } \\
\text { dental care: aseries of cases } \\
\text { (Macedo et al., 2019) }\end{array}$ & $\begin{array}{l}\text { Relato de serie } \\
\text { decasos }\end{array}$ & $\begin{array}{l}\text { Local: Hospital UniversitárioPernambuco-Brasil } \\
\text { Intervenção } \quad \text { odontológica realizada: } \\
\text { atuação do cirurgião- dentista em ambiente } \\
\text { hospitalar, participando como membro ativo do } \\
\text { uma equipe multidisciplinar em oncologia em } 3 \\
\text { casos de pacientes oncológicos }\end{array}$ & $\begin{array}{l}\text { A presença de um cirurgião-dentista atuante na } \\
\text { equipe multiprofissional em oncologia atua na } \\
\text { prevenção, diagnóstico e tratamento das afecções } \\
\text { bucais e efeitos colaterais da terapia antineoplásica, } \\
\text { a MO, levando a uma melhora nas condições } \\
\text { sistêmicas e qualidade de vida de pacientes } \\
\text { hospitalizados }\end{array}$ & $\begin{array}{l}\text { É responsabilidade do CD e equipe oncológica } \\
\text { multiprofissional participar das ações de } \\
\text { promoção à saúde, diagnóstico, controle e } \\
\text { tratamento das repercussões orais e dentais, e } \\
\text { manter o seguimento de longo prazo, } \\
\text { contribuindo para a melhora da condição bucal e } \\
\text { sistêmica, bem como qualidade de vida dos } \\
\text { pacientes, proporcionando um tratamento } \\
\text { integral e humanizado }\end{array}$ \\
\hline
\end{tabular}


Research, Society and Development, v. 10, n. 4, e33410414117, 2021

(CC BY 4.0) | ISSN 2525-3409 | DOI: http://dx.doi.org/10.33448/rsd-v10i4.14117

\begin{tabular}{|c|c|c|c|c|}
\hline $\begin{array}{l}\text { Dental care pathways for adult } \\
\text { in patientsin an acute } \\
\text { hospital: a five-year service } \\
\text { evaluation (Hashem et al., } \\
\text { 2020) }\end{array}$ & $\begin{array}{l}\text { Estudo } \\
\text { transversal } \\
\text { retrospectivo }\end{array}$ & $\begin{array}{l}\text { Local: East Surrey Hospital } \\
\text { - Inglaterra } \\
\text { Período: 01/2014 a 12/2018 } \\
\text { Amostra: } 851 \text { pacientes que foram admitidos } \\
\text { no hospital durante o período } \\
\text { Coleta de dados: } \\
\text { Foram avaliadas as razões para o } \\
\text { encaminhamento para dentista e serviço } \\
\text { maxilofacial e os benefícios dos tratamentos } \\
\text { realizados }\end{array}$ & $\begin{array}{l}851 \text { pacientes foram recebidas para avaliação } \\
\text { equipe odontológica no período estudado e os } \\
\text { tratamentos da equipe foram desde aconselhamento } \\
\text { até extração dos dentes; } \\
\text { Relataram que o tratamento dentário fez com que o } \\
\text { paciente voltasse a comer após úlcera causada no } \\
\text { dente ajudou a reduzir o risco de complicações de } \\
\text { problemas cardíacos e reduzir a dependência no } \\
\text { alívio da } \\
\text { dor hospitalar }\end{array}$ & $\begin{array}{l}\text { Existem ligações entre saúde bucal deficiente e } \\
\text { saúde geral, e como isto tem impacto global } \\
\text { sobre os doentes internadosem hospitais; } \\
\text { Intervenções pequenas e eficazes podem levar a } \\
\text { melhores resultados aos pacientes; } \\
\text { Este estudo mostrou que o hospital pode utilizar } \\
\text { as competências da equipe odontológica, com } \\
\text { objetivo de preservar a dignidade do paciente e } \\
\text { saúde e melhorar ou reduzir a internação dos } \\
\text { pacientes }\end{array}$ \\
\hline $\begin{array}{l}\text { Avaliação odontológica de } \\
\text { pacientes em unidade de } \\
\text { terapia intensiva (UTI) } \\
\text { pediátrica } \\
\text { (Austriaco-Leite et al., 2018) }\end{array}$ & $\begin{array}{l}\text { Estudo } \\
\text { descritivo } \\
\text { retrospectivo, } \\
\text { transversal }\end{array}$ & $\begin{array}{l}\text { Local: UTI Pediátrica Hospital Maranhão - } \\
\text { Brasil; } \\
\text { Amostra: pacientes de } 0 \text { a } 15 \text { anos; Período: } \\
\text { 09/2016 a 11/2017; } \\
\text { Coleta de dados: Foram analisados motivo da } \\
\text { internação, condição das vias aéreas, doença } \\
\text { bucal, procedimentos odontológicos } \\
\text { realizados, higiene bucal e presençade pneumonia } \\
\text { associada à ventilação mecânica, sendo } \\
\text { que todos os pacientes recebiam higiene bucal } \\
\text { diariamente }\end{array}$ & $\begin{array}{l}45,83 \% \text { dos pacientes apresentavam higiene bucal } \\
\text { satisfatória, } 45,14 \% \text { regular e } 9,03 \% \text { deficiente; } \\
\text { Em relação às condições da mucosa bucal, apenas } \\
5,52 \% \text { dos pacientes apresentaram alterações, } \\
\text { destas, } 3,45 \% \text { já se encontravam no momento da } \\
\text { admissão; } \\
\text { Foram tratados } 8 \text { pacientes com lesões bucais e } \\
\text { realizadas } 6 \text { exodontias; } \\
\text { O acompanhamento odontológico diário na UTI } \\
\text { garantiu a rotina de higiene bucal e manutenção de } \\
\text { uma adequada condição bucal }\end{array}$ & $\begin{array}{l}\text { Este estudo revelou que crianças hospitalizadas } \\
\text { em UTI Pediátrica podem apresentar lesões em } \\
\text { mucosa bucal desde a admissão ou logo após a } \\
\text { internação, sendo necessário a presença do CD na } \\
\text { restauração da saúde bucal destes pacientes e } \\
\text { prevenindo eventos adversos na cavidade bucal, } \\
\text { que podem agravar e retardar a recuperação do } \\
\text { paciente, visando a menor permanência no } \\
\text { hospital e o atendimento em sua integralidade; } \\
\text { O CD exerce importante função na equipe, para } \\
\text { garantir atenção integral aos indivíduos, } \\
\text { minimizando problemas } \\
\text { ocasionados por alterações bucais esistêmicas }\end{array}$ \\
\hline $\begin{array}{l}\text { Review of a three-year study } \\
\text { on the dental care of onco- } \\
\text { hematological pediatric } \\
\text { patients }\end{array}$ & $\begin{array}{l}\text { Estudo } \\
\text { Transversal }\end{array}$ & $\begin{array}{l}\text { Período:01/2015 a 12/2017 } \\
\text { Local: Hospital São Paulo -Brasil } \\
\text { Coleta dedados: Prontuários médicos e } \\
\text { odontológicos de pacientes atendidos na }\end{array}$ & $\begin{array}{l}\text { Foram realizados } 1411 \text { procedimentos } \\
\text { odontológicos: } \\
\text { odontológico preventivo }(33 \%) ; \\
\text { Cirurgias bucomaxilofaciais }(26 \%) \text {; Odontologia }\end{array}$ & $\begin{array}{l}\text { Esse resultado enfatiza a importância do } \\
\text { atendimento multiprofissional, incluindo o CD, } \\
\text { na redução da dor e desconforto do paciente } \\
\text { hospitalizado }\end{array}$ \\
\hline
\end{tabular}


Research, Society and Development, v. 10, n. 4, e33410414117, 2021

(CC BY 4.0) | ISSN 2525-3409 | DOI: http://dx.doi.org/10.33448/rsd-v10i4.14117

\begin{tabular}{|c|c|c|c|c|}
\hline (Frascino et al., 2018) & & $\begin{array}{l}\text { unidadeodontológica do serviço } \\
\text { oncohematologia do Instituto da Criança; } \\
\text { Amostra: } 565 \text { pacientes: bebês, crianças ou } \\
\text { adolescentes ( } 0 \text { a } 18 \text { anos) diagnosticados com } \\
\text { doença onco-hematológica }\end{array}$ & $\begin{array}{l}\text { restauradora (14\%) e a aplicação de laser tópico } \\
\text { para MO }(11 \%) ; \quad \text { periodontite e endodontia } \\
(15 \%) ; \\
\text { Procedimentos preventivos reduziram a frequência } \\
\text { do tratamento restaurador e prevalência de focos } \\
\text { infecciosos; } 11 \% \text { dos protocolos odontológicos } \\
\text { consistiam em aplicações tópicas delaser }\end{array}$ & \\
\hline $\begin{array}{l}\text { Multicenter phase II study of } \\
\text { an oral care program for } \\
\text { patients with head and neck } \\
\text { cancer receiving } \\
\text { chemoradiotherapy (Yokota et } \\
\text { al., 2016) }\end{array}$ & $\begin{array}{l}\text { Estudo } \\
\text { longitudinal } \\
\text { observacional }\end{array}$ & $\begin{array}{l}\text { Periodo: 04/20012 a 12/2013 } \\
\text { Local: Japão } \\
\text { Amostra: Pacientes com câncer de cabeça e } \\
\text { pescoço, de } 20-75 \text { anos, recepção programada de } \\
\text { irradiação para o espaço bucal; com capacidade } \\
\text { alimentar e normalidade da dieta, ausência de } \\
\text { MO, em doentes com cancro da cabeça e } \\
\text { pescoço; } \\
\text { Intervenção odontológica realizada: Os } \\
\text { pacientes recebiam avaliação odontológica e } \\
\text { tratamentodentário; foram realizadas ao longoda } \\
\text { quimioradioterapia instruções de higiene, } \\
\text { cuidados bucais e avaliados MO; } \\
\text { acompanhamento para verificar toxicidade tardia } \\
\text { relacionada com radioterapia até } 1 \text { ano após a } \\
\text { conclusão }\end{array}$ & $\begin{array}{l}\text { Sessenta e três pacientes }(52,5 \%) \text { desenvolveram } \\
\text { MO de grau } 3 \text { (funcional/sintomático) e um }(0,8 \%) \\
\text { desenvolveu MO de grau 4; } \\
70 \text { pacientes }(58,3 \%) \text { não conseguiram obter uma } \\
\text { nutrição adequada por via oral, } 14 \text { deles não foram } \\
\text { capazes de obter uma nutrição suficiente através } \\
\text { ingestão oral apesar de inferior ao grauMO grau } 2 \\
\text { devido a outras complicaçães; } \\
\text { A incidência de MO grau } \leq 1 \text { a } 2 \text { e } 4 \text { semanas após } \\
\text { a conclusão da radioterapia foi de } 34,2 \text { e } 67,6 \% \text {, } \\
\text { respectivamente; entretanto, } 24,8 \text { e } 6,3 \% \text { dos } \\
\text { pacientes, respectivamente, ainda tinham MO grau } \\
3 \text {. } \\
\text { A radioterapia foi concluída em 99,2\% dos } \\
\text { doentes (119/120) sendo que um paciente foi } \\
\text { programado para } 70 \text { Gy de radiação, mas parou aos } \\
60 \text { Gy por causa da MO grau } 4\end{array}$ & $\begin{array}{l}\text { Era esperado que o programa de cuidados bucais } \\
\text { reduzisse a incidência de mucosite induzida por } \\
\text { quimioradioterapia, mas os resultados revelaram } \\
\text { que o MO grau } \geq 3 \text { (funcional/sintomático) } \\
\text { ocorreu em mais demetade dos doentes; } \\
\text { Um programa sistemático de cuidados bucais por } \\
\text { si só é insuficiente para diminuira incidência de } \\
\text { MO grave induzida por quimioterapia; } \\
\text { Estes resultados sugerem que os programas de } \\
\text { cuidados bucais podem melhorar indiretamente o } \\
\text { cumprimento do tratamento por meio da redução } \\
\text { do risco deinfecção; o programa de higiene bucal } \\
\text { durante e após a radioterapia pode diminuir o } \\
\text { risco de complicações dentárias tardias, } \\
\text { incluindo a osteorradionecrose e ajudam a } \\
\text { reduzir as consequências nutricionais a longo } \\
\text { prazo, prevenindo toxicidade tardia }\end{array}$ \\
\hline $\begin{array}{l}\text { Continuous } \text { Professional Oral } \\
\text { Health Care Intervention } \\
\text { Improves Severe Aspiration } \\
\text { Pneumonia }\end{array}$ & Estudo de caso & $\begin{array}{l}\text { Local: Japão } \\
\text { Intervenção } \\
\text { O Cuidado por profissionais de Saúde Bucal } \\
\text { (CPSB) foi solicitado pelo médico e a família do }\end{array}$ & $\begin{array}{l}\text { No início, com a intervenção CPSB, oestado bucal } \\
\text { do paciente piorou e ficou seco durante } 1 \text { dia, com } \\
\text { vermelhidão na mucosa bucal e dorso da língua } \\
\text { após remoção de substâncias membranosas, com }\end{array}$ & $\begin{array}{l}\text { A pneumonia não melhorou até começar a } \\
\text { intervenção do CPSB; a intervenção persistente } \\
\text { contribuiu para diminuição da gravidade da } \\
\text { pneumonia; }\end{array}$ \\
\hline
\end{tabular}




\begin{tabular}{|c|c|c|c|c|}
\hline (Nawata et al., 2019) & & $\begin{array}{l}\text { doente para melhorar condição bucal: Foi } \\
\text { realizada a limpeza duas vezes por dia utilizando } \\
\text { escovasdescartáveis para remoção de substâncias } \\
\text { membranosas e gel hidratante que também } \\
\text { forneceu umidade estabilizando a mucosa bucal e } \\
\text { dorso da língua }\end{array}$ & $\begin{array}{l}\text { dor aparente, apesar do procedimento suave; } \\
\text { Os cuidados persistiram e tornaram-se } \\
\text { gradualmente eficaz para uma condição bucal } \\
\text { saudável em } 2 \text { semanasapós o início da intervenção, } \\
\text { sem dor evermelhidão; } \\
\text { Após a recuperação saúde bucal, a febre do doente } \\
\text { diminuiu e proteína CReativa (indicativo de } \\
\text { processos inflamatórios ou infecciosos) voltaram } \\
\text { aos níveis normais, melhorando anormalidade } \\
\text { de raios X e consciência }\end{array}$ & $\begin{array}{l}\text { Foi sugerido que a remoção mecânica dos } \\
\text { agentes patogénicos bucais pode impedir a } \\
\text { exacerbação da pneumonia por aspiração em } \\
\text { doentes com disfagia, não obstante os efeitos dos } \\
\text { antibióticos; } \\
\text { O CPSB pode reduzir o número de bactérias } \\
\text { orofaríngeas, incluindo agentes } \\
\text { patogénicos respiratórios a estimulaçãomecânica } \\
\text { da mucosa bucal, que leva à estimulação do } \\
\text { cérebro e melhorias em disfagia, tempo de } \\
\text { latência da resposta de deglutição e o reflexo da } \\
\text { tosse }\end{array}$ \\
\hline $\begin{array}{l}\text { Is it necessary to have a } \\
\text { dentist within anintensive care } \\
\text { unit team? Report of a } \\
\text { randomisedclinical trial } \\
\text { (Bellissimo-Rodrigues et al., } \\
2018 \text { ) }\end{array}$ & $\begin{array}{l}\text { Ensaio clínico } \\
\text { randomizado } \\
\text { Experimental }\end{array}$ & $\begin{array}{l}\text { Período: 01/2011 a 08/2013 } \\
\text { Local: São Paulo - Brasil } \\
\text { Amostra: pacientes adultos internados na UTI } \\
\text { durante } 48 \text { horas ou mais, divididos em grupo } \\
\text { experimental ( } \mathrm{n}=127 \text { ) comtratamento dentário } 4 \text { a } \\
5 \text { vezes por semana, além da higiene bucal de } \\
\text { rotina e grupo de controle ( } \mathrm{n}=127 \text { ) com higiene } \\
\text { bucal de rotina (aplicação tópica de clorexidina) } \\
\text { fornecida pela enfermagem da UTI }\end{array}$ & $\begin{array}{l}\text { O estado de saúde bucal de base dos pacientes era } \\
\text { pobre e incluía edentulismo, cárie, gengivite, } \\
\text { periodontite e raízes residuais; } \\
\text { Os resultados do Índice de Higiene Oral } \\
\text { Simplificado e do Índice Gengival diminuíram no } \\
\text { grupo experimental, mas não se alteraram } \\
\text { significativamente no grupo de controle durante a } \\
\text { estadia na UTI; } \\
\text { O tratamento dentário evitou a maior parte dos } \\
\text { episódios de infecções do trato respiratório }\end{array}$ & $\begin{array}{l}\text { Numa perspectiva interprofissional, os resultados } \\
\text { apoiam a ideia de incluir dentistas na equipe da } \\
\text { UTI para melhorar a saúde bucal em pacientes } \\
\text { críticos e prevenir eficazmente as infecções do } \\
\text { tratorespiratório }\end{array}$ \\
\hline $\begin{array}{l}\text { Upper aerodigestive tract } \\
\text { cancer and oral health status } \\
\text { before } \\
\text { radiotherapy: A cross- } \\
\text { sectional study of154 patients }\end{array}$ & $\begin{array}{l}\text { Estudo } \\
\text { transversal } \\
\text { observacional }\end{array}$ & $\begin{array}{l}\text { Local: departamento Odontologia Hospital } \\
\text { Marselha - França Amostra: } \\
\text { dentados agendados para radioterapia, } \\
\text { commais de } 18 \text { anos, sem deficiência. Coleta de } \\
\text { dados: } 154 \text { pacientes com tumor submetidos a } \\
\text { avaliação bucal por dois dentistas, realizadas }\end{array}$ & $\begin{array}{l}\text { Os locais mais comuns de tumores foram a laringe } \\
(28,6 \%) \text { e a cavidade bucal }(26,6 \%) \text {, pacientes } \\
\text { fumantes }(80,5 \%) \text { e que consumiam bebidas } \\
\text { alcoólicas }(67 \%) \text {; com higiene bucal inadequada; } \\
\text { O nível ósseo foi de } 62,3 \% \text { e } 57,8 \% \text { dos pacientes } \\
\text { com infecções ósseas, que se encontram ou são }\end{array}$ & $\begin{array}{l}\text { Pacientes com câncer no trato aerodigestivo são } \\
\text { um grupo de alto risco para osteonecrose se o } \\
\text { tratamento e cuidados dentários não forem } \\
\text { prestados antesradioterapia; } \\
\text { É necessária mais investigação paradeterminar se } \\
\text { o plano de tratamento adotado antes da }\end{array}$ \\
\hline
\end{tabular}


Research, Society and Development, v. 10, n. 4, e33410414117, 2021

(CC BY 4.0) | ISSN 2525-3409 | DOI: http://dx.doi.org/10.33448/rsd-v10i4.14117

\begin{tabular}{|c|c|c|c|c|}
\hline (Raskin et al., 2018) & & $\begin{array}{l}\text { radiografias, com realização do tratamento } \\
\text { necessário }\end{array}$ & comuns coma falta de higiene $(\mathrm{p}=0,04)$ & $\begin{array}{l}\text { radioterapia foi bem- sucedido em manter uma } \\
\text { boa saúde bucal } \\
\text { nesta população e para estabelecer um consenso } \\
\text { de boas práticas. }\end{array}$ \\
\hline 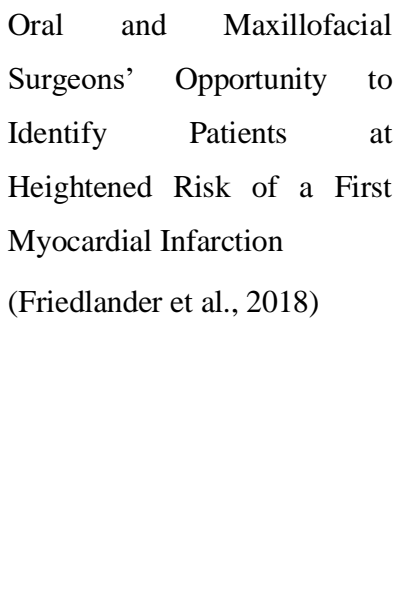 & $\begin{array}{l}\text { Estudo Caso- } \\
\text { controle } \\
\text { observacional }\end{array}$ & $\begin{array}{l}\text { Periodo: } 2018 \\
\text { Local: Universidades Umea e Karolinska na } \\
\text { Suécia } \\
\text { Intervenção odontológica realizada: } \\
\text { Análise de imagens panorâmicas por CD e Buco- } \\
\text { maxilofacial que tiveram as imagens obtidas } 6 \text { a } \\
10 \text { semanas após a manutenção de um infarto de } \\
\text { miocárdio comparados com } \\
\text { imagens de controles saudáveis sem } \\
\text { históricocardiovascular semelhantes ao grupo } \\
\text { experimental }\end{array}$ & $\begin{array}{l}\text { Entre os casos masculinos }(\mathrm{n}=562 \text {; idade média, } \\
61 \text { anos), } 32,7 \% \text { evidenciaram placas calcificadas } \\
\text { da artéria carótida unilateral ou bilateral na sua } \\
\text { imagem panorâmica contra } 26,5 \% \text { do grupo } \\
\text { controle (OR } 1,235 ; \mathrm{p}=0.022) ; \\
\text { Entre os casos femininos ( } \mathrm{n}=134 \text {; idade média, } 64 \\
\text { anos), } 21,6 \% \text { de da artéria carótida bilateral versus } \\
11,9 \% \text { de grupo controle (OR } 1,99 ; \mathrm{p}=0.047)\end{array}$ & $\begin{array}{l}\text { Com avaliação de imagens panorâmicas, durante } \\
\text { anamnese é possível identificar indivíduos } \\
\text { assintomáticos com arteroesclerose } \\
\text { que, estão em risco de infarto do miocárdio a } \\
\text { curto prazo }\end{array}$ \\
\hline
\end{tabular}

Fonte: Autores. 


\section{Discussão}

Esta revisão de escopo mapeou os principais benefícios da intervenção odontológica do cirurgião-dentista em ambiente hospitalar da literatura e mostrou sua importância para os pacientes com a prevenção de doenças, melhoria na qualidade de vida, bem como para o hospital com a diminuição do tempo de internação e com redução de custos.

Neste estudo foram identificados diversos benefícios que o cirurgião dentista pode contribuir tanto para os pacientes, quanto para os hospitais, que foram detalhados nas próximas sessões.

\section{Prevenção de infarto do miocárdio}

O infarto agudo do miocárdio (IAM) é a primeira causa de mortes no Brasil (Brasil, 2017). O custo dos procedimentos que integram o tratamento do IAM é bem alto, em torno de R\$12.873,69 a 23.461,87 (Friedlander et al., 2018).

Estudo realizado na Suécia, longitudinal caso-controle, que analisou radiografias panorâmicas de pacientes que tiveram infarto em comparação aos que não tiveram, mostrou a importância do $\mathrm{CD}$ ao avaliar radiografias panorâmicas, a fim de identificar a presença de placas calcificadas na artéria carótida, considerado neste estudo, indício que o paciente pode sofrer um infarto do miocárdio futuramente (Friedlander et al., 2018).

\section{Prevenção da mucosite oral}

A MO é um dos eventos adversos da radioterapia e quimioradioterapia e deteriora a qualidade de vida dos doentes, pois causa dores substanciais, interferindo na capacidade dos pacientes de mastigar e comer, possivelmente o levando à desnutrição. A irradiação também danifica as glândulas salivares, o que causa secura de boca e facilita a proliferação bacteriana no interior da cavidade bucal. Além disso, os agentes quimioterapêuticos podem induzir mielossupressão (atividade diminuída da medula óssea, resultando em menor número de glóbulos vermelhos, glóbulos brancos e plaquetas) e, assim agravar a MO. Estes fatores aumentam a susceptibilidade do doente não só à infecção bucal, mas também à PAVM (Yokota et al., 2016).

Macedo et al. (2019) sugerem que a presença do CD na equipe multidisciplinar do hospital pode atuar na prevenção da mucosite oral em pacientes oncológicos, antes de seu aparecimento. Franscino et al (2018), em seu estudo transversal em São Paulo-Brasil, mostra a importância do atendimento do CD, na redução da dor e desconforto do paciente hospitalizado em relação a mucosite oral. Porém, Yokota et al (2016) realizaram um estudo observacional com pacientes com câncer de cabeça e pescoço e verificaram que o programa sistemático de higiene bucal que foi proposto foi insuficiente para diminuir a incidência de MO grave nestes pacientes em tratamento com quimiorradioterapia, porém pode trazer benefícios de forma indireta.

\section{Prevenção de osteorradionecrose}

A osteorradionecrose é uma complicação grave da radioterapia (utilizada para o tratamento das neoplasias de cabeça e pescoço), na qual o osso irradiado torna-se desvitalizado e exposto pela perda da integridade da pele e da mucosa, persistindo sem cicatrização. Já é sabido na literatura que alguns problemas bucais como periodontopatias e extrações dentárias são fatores de risco para esta doença (Aldunate et al., 2010).

Raskim et al. (2017), em seu estudo transversal na França, sugeriram que pacientes com câncer no trato aerodigestivo são um grupo de alto risco para osteonecrose se o tratamento odontológico não for prestado antes radioterapia. Macedo, Melo e Vidal (Macedo et al., 2019) e Yokota et al.(Yokota et al., 2016) também sugeriram que a intervenção odontológica age na prevenção da osteorradionecrose. 


\section{Prevenção da Pneumonia}

A pneumonia associada à ventilação mecânica (PAVM) é a infecção que acomete o parênquima pulmonar de pacientes em uso do ventilador mecânico por mais de $48 \mathrm{hs}$ e que não tiveram a ventilação como motivo de entubação (Cardoso \& Bizani, 2015). A PAVM é a segunda infecção mais frequente nas UTIs americanas e a mais frequente em UTIs européias, possui altos índices de mortalidade associada e altos custos relacionados a maior tempo de internação e uso de antimicrobianos. No Brasil, há ausência de dados nacionais e multicêntricos, mas experiências individuais traz que a PAVM é uma das mais frequentes infecções na UTI (Camargo \& Grinbaum, 2006).

Sato et al. (2016), em estudo observacional no Japão, sugerem que os dentistas devem avaliar e realizar atendimento odontológico nos pacientes para redução da probabilidade de pneumonia grave após a esofagectomia. Nawata et al. (2019), no estudo de caso realizado no Japão, sugeriram que os as condutas realizadas por profissionais de saúde bucal contribuíram efetivamente para a diminuição da gravidade da pneumonia, com estimulação do cérebro e melhorias em disfagia, tempo de latência da resposta de deglutição e o reflexo da tosse. Este artigo não deixa claro como é composta esta equipe de profissionais de saúde bucal.

\section{Prevenção de infecção e outras doenças oportunistas}

Yokota et al. (2016) trouxeram que programas sistemáticos de higiene bucal podem melhorar indiretamente a adesão ao tratamento, diminuindo o risco de infecção. Belíssimo-Rodrigues et al. (2018), em seu estudo clínico randomizado, concluem que dentistas na equipe da UTI pode melhorar a saúde bucal em pacientes críticos e prevenir eficazmente as infecções do trato respiratório. Frascino et al. (2018) sugerem que procedimentos odontológicos preventivos, como aplicação tópica de flúor e higiene bucal profissional reduziram a frequência do tratamento restaurador e a prevalência de focos infecciosos, e enfatizam que $C D$ deve ser incluído na equipe multiprofissional, coma finalidade de redução da dor e desconforto do paciente hospitalizado que recebem terapias antineoplásicas(Frascino et al., 2018).

\section{Diminuição tempo de internação e custo para Hospital}

Estudo realizado no EUA mostrou que a redução de PAVM chegou a 46\% com a odontologia hospitalar (Sona et al., 2009).

Hashem, Gillway e Doshi (2020), em seu estudo transversal na Inglaterra, mostraram a importância de o hospital utilizar as competências do cirurgião-dentista para o tratamento dos pacientes, a fim de melhorar ou reduzir a internação dos pacientes.

Austríaco-Leite et al (2018) trouxeram, em seu estudo transversal, que a presença do cirurgião-dentista no tratamento para a restauração da saúde bucal de pacientes pediátricos, com prevenção de eventos adversos na cavidade bucal, pode acelerar a recuperação do paciente, visando a menor permanência no hospital e realizando o atendimento em sua integralidade.

Belíssimo-Rodrigues et al. (2018), apesar de não terem realizado análise de custo-benefício em seu estudo, sugerem que a inclusão de um cirurgião dentista na equipe de tratamento da UTI pode ser uma opção econômica, visto que cada caso de PAVM pode causar um custo extra e significante para o hospital.

\section{Melhoria na condição sistêmica do paciente e qualidade de vida}

Miranda e Montenegro (2010), estudo de caso no Distrito Federal-Brasil, traz que a inserção do CD no hospital pode ajudar na mudança de quadros clínicos odontológicos prejudiciais à saúde sistêmica. Os estudos de alguns autores (Frascino et al., 2018; Hashem et al., 2020; Macedo et al., 2019; Nawata et al., 2019; Yokota et al., 2016) apontam a melhoria da qualidade de vida dos pacientes após intervenções odontológicas. 
As intervenções odontológicas citadas neste estudo deixam claro as vantagens da inserção do CD na equipe hospitalar. Dentre esses procedimentos, são realizadas raspagens sub e supra gengivais, análise de exames radiográficos panorâmicos, extrações dentarias, profilaxia, entre outros. Somente em um estudo, que foi incluído na revisão, não ficou claro a participação do CD na intervenção, pois eles mencionaram apenas cuidados realizados por profissionais de saúde bucal (Nawata et al., 2019). A participação de dentistas, de forma mais ativa, nos serviços realizados em nível ambulatorial ou hospitalar, tem o propósito de auxiliar, oferecer e agregar mais qualidade ao atendimento prestado em ambiente hospitalar, dando maior destaque na integralidade da atenção e assistência (Aldunate et al., 2010).

Belíssimo-Rodrigues et al (2018), fez um estudo comparando o atendimento bucal em pacientes realizado por um dentista (grupo teste) com o realizado por uma enfermeira (grupo controle). $\mathrm{O}$ atendimento realizado pelo dentista consistiu em na escovação de dentes, raspagem da língua, remoção do cálculo, descamação e aplainamento radicular, tratamento restaurador atraumático de cárie e extração de dentes, de acordo com a necessidade do paciente. Enquanto o grupo atendido pela enfermeira teve acesso apenas ao protocolo de higiene bucal de rotina, que consiste na limpeza mecânica da cavidade oral com uma espátula envolvida em gaze, seguida da aplicação tópica de $0.12 \%$ de solução de clorexidina ou 2,0\% de gel de clorexidina, de acordo com o nível de consciência do doente. As evidências deste estudo mostraram que embora os profissionais de enfermagem da UTI desempenhem um papel satisfatório na promoção da higiene bucal, os resultados indicaram que os pacientes tratados por dentistas tinham melhores resultados. Portanto, esse estudo apoia a ideia de incluir um dentista na equipe de cuidados intensivos para melhorar o estado de saúde bucal de doentes críticos, para além da melhoria possível pelo pessoal da enfermagem.

A Odontologia Hospitalar no Brasil avançou nas últimas décadas. Em 2008 foi feito o projeto de Lei 2776/2008 (Brasil, 2019a) que objetivava a inserção obrigatória do cirurgião-dentista em UTI, clínicas, hospitais públicos e privados. Porém, em 05 de Junho de 2019, ele foi vetado (Brasil, 2019b). Estudos, como esta revisão de escopo, ressaltam a importância da inserção do CD em ambiente hospitalar, trazendo a necessidade de impulsionar a discussão sobre a temática, principalmente pelos resultados e benefícios positivos para a população e para o estabelecimento de saúde.

O estudo teve resultados interessantes e significantes, porém precisam ser considerados à luz das suas limitações. Apesar dos autores terem planejado cuidadosamente as estratégias de buscas, consultando Descritores em Ciências da Saúde, realizando testes com diferentes descritores para escolher aqueles mais apropriados, existe a possibilidade de que alguns artigos científicos publicados não tenham sido identificados nesta revisão. Há necessidade de ampliar a busca em maior número de bases de dados para verificar que se há outros benefícios ou maior qualidade das evidências. Outro ponto negativo foi a inclusão de estudos com baixa evidência, ressaltando a escassa literatura na área, apontando para a necessidade de estudos futuros, com metodologias e amostras mais robustas.

Esta revisão de escopo tem pontos positivos que merecem ser destacados, principalmente pela síntese dos principais benefícios da inserção do cirurgião-dentista para a população, hospitais e redução de custos para a saúde pública. Estes resultados devem ser levados em conta para contratação de profissionais desta área e planejamento de ações dentro do hospital para que haja a promoção de saúde dos pacientes internados. Este profissional servirá como uma base no diagnóstico das condições bucais, tratamento odontológico, aliado a terapêutica médica, proporcionando maior conforto do paciente e para terem o meio bucal satisfatório.

\section{Considerações Finais}

Os resultados sugerem que são variados e valiosos os benefícios que um profissional da Odontologia intervindo no ambiente hospitalar traz para a população e o estabelecimento de saúde, tais como: prevenção de pneumonia, focos 
infecciosos, osteorradionecrose, infarto do miocárdio; redução de mucosite oral no tratamento de câncer, com melhoria da qualidade de vida; diminuição da internação e custo hospitalar. Assim, a presença do cirurgião-dentista neste local deve ser estimulada, sendo um importante elemento na equipe interprofissional hospitalar, a fim de proporcionar além de ganhos já citados, mas também para a redução de custos para a saúde pública.

A escassa literatura de estudos nesta área direciona a necessidade de realização de estudos primários, com metodologias bem delineadas e com amostras robustas para evidenciar achados mais consistentes sobre o assunto estudado.

\section{Agradecimentos}

Á Pró-Reitoria de Pesquisa da UFMG pela Iniciação Científica.

\section{Referências}

Aldunate, J. L., Aldunate, B., Coltro, P. S., Busnardo, F. F., \& Ferreira, M. C. (2010). Osteoradionecrosis in face: pathophysiology, diagnosis and treatment. Revista Brasileira Cirurgia Plástica, 25(2), 381- 387.

AranegaI, A. M., BassiI, A. P. F., PonzoniI, D., WayamaII, M. T., Esteves, J. C., \& Garcia-Junior, I. R. (2012). Qual a importância da Odontologia Hospitalar? Revista Brasileira Odontologia, 69, 90-92. Arksey, H., \& O'Malley, L. (2005). Scoping studies: towards a methodological framework. International Journal of Social Research Methodology, 8(1), 19-32.

Austriaco-Leite, H. L., Ferreira-Lopes, F., Silva, M. d. S. A. C., \& Diniz-Souza, L. C. (2018). Avaliação odontológica de pacientes em unidade de terapia intensiva (UTI) pediátrica. CES Odontologia, 31(2), 6-14.

Bellissimo-Rodrigues, W. T., Menegueti, M. G., Gaspar, G. G., de Souza, H. C. C., Auxiliadora-Martins, M., Basile-Filho, A., Martinez, R., \& BellissimoRodrigues, F. (2018). Is it necessary to have a dentist within an intensive care unit team? Report of a randomised clinical trial. Int Dent J, 68(6), $420-427$.

Blum, D. F. C., Silva, J. A. S., Baeder, F. M., \& Bona, Á. D. (2018). The practice of dentistry in intensive care units in Brazil. Revista Brasileira Terapia Intensiva, 30(3), 327-332.

Brasil. (2017). Brasil. Taxa de mortalidade - DATASUS http://svs.aids.gov.br/dantps/centrais-de- conteudos/paineis-de-monitoramento/mortalidade/gbdbrasil/principais-causas/

Brasil. Projeto de Lei 883/19 - Obrigatoriedade da presença de profissionais de odontologia nas unidades de terapia intensiva. (2019a). https://www.camara.leg.br/proposicoesWeb/fichadetramitacao?idProposicao=2192355

Brasil. Veto $\mathrm{n}^{\mathrm{o}} \quad 16 / 2019$. (2019b).

Camargo, L. F. A., \& Grinbaum, R. S. (2006). Diretrizes sobre Pneumonia Associada à Ventilação Mecânica (PAVM).

Cardoso, M. E. V., \& Bizani, D. (2015). Aplicação de bundle de prevenção de pneumonia associada à ventilação mecânica em centro de terapia intensiva adulto: um relato de experiência. Saúde Desenvolvimento Humano, 3(2), 137-146.

DeRiso, A. J., Ladowski, J. S., Dillon, T. A., Justice, J. W., \& Peterson, A. C. (1996). Chlorhexidine gluconate $0.12 \%$ oral rinse reduces the incidence of total nosocomial respiratory infection and nonprophylactic systemic antibiotic use in patients undergoing heart surgery. Chest, 109(6), $1556-1561$.

Frascino, A. V., Fava, M., Cominato, L., \& Odone-Filho, V. (2018). Review of a three-year study on the dental care of onco-hematological pediatric patients. Clinics (Sao Paulo), 73, e721.

Friedlander, A. H., Lee, U. K., Chang, T. I., \& Bostrom, K. I. (2018). Oral and Maxillofacial Surgeons' Opportunity to Identify Patients at Heightened Risk of a First Myocardial Infarction. J Oral Maxillofac Surg, 76(10), 2041-2043.

Gaetti-Jardim, E., Setti, J. S., Cheade, M. d. F. M., \& Mendonça, J. C. G. (2013). Atenção odontológica a paciente hospitalizados: revisão da literatura e proposta de protocolo de higiene oral. Rev. Bras. Cienc. saude. Revista Brasileira Ciências Saude, 11 (35), 31-36.

Hashem, I. W., Gillway, D., \& Doshi, M. (2020). Dental care pathways for adult inpatients in an acute hospital: a five-year service evaluation. Br Dent J, 228(9), 687-692.

Macedo, T. S., Melo, M. C. F., \& Vidal, A. K. d. L. (2019). Hospital and oncological dental care: a series of cases. Revista Gaucha Odontologia, 67(e20190036).

Miranda, A. F., \& Montenegro, F. L. B. (2010). Ação odontológica preventiva em paciente idoso dependente na Unidade de terapia Intensiva (UTI): relato de caso. Revista Paulista Odontologia, 32(1), 34-38.

Nawata, W., Umezaki, Y., Yamaguchi, M., Nakajima, M., Makino, M., Yoneda, M., Hirofuji, T., Yamano, T., Ooboshi, H., \& Morita, H. (2019). Continuous Professional Oral Health Care Intervention Improves Severe Aspiration Pneumonia. Case Rep Dent. 
Research, Society and Development, v. 10, n. 4, e33410414117, 2021

(CC BY 4.0) | ISSN 2525-3409 | DOI: http://dx.doi.org/10.33448/rsd-v10i4.14117

Rabelo, G. D., Queiroz, C. I., \& Santos, P. S. S. (2010). Atendimento odontológico ao paciente em unidade de terapia intensiva. Arquivos Medicos Hospital Ciencias Medicas Santa Casa São Paulo, 55(2), 67-70.

Raskin, A., Ruquet, M., Weiss-Pelletier, L., Mancini, J., Boulogne, O., Michel, J., Fakhry, N., Foletti, J. M., Chossegros, C., \& Giorgi, R. (2018). Upper aerodigestive tract cancer and oral health status before radiotherapy: A cross-sectional study of 154 patients. J Stomatol Oral Maxillofac Surg, $119(1), 2-7$.

Sato, Y., Motoyama, S., Takano, H., Nakata, A., Liu, J., Harimaya, D., Todo, N., Yoshino, K., Sasaki, T., Wakita, A., Kawakita, Y., Imai, K., Saito, H., Fukuda, M., \& Minamiya, Y. (2016). Esophageal Cancer Patients Have a High Incidence of Severe Periodontitis and Preoperative Dental Care Reduces the Likelihood of Severe Pneumonia after Esophagectomy. Digestive surgery, 33(6), 495- 502.

Sona, C. S., Zack, J. E., Schallom, M. E., McSweeney, M., McMullen, K., Thomas, J., Coopersmith, C. M., Boyle, W. A., Buchman, T. G., Mazuski, J. E., \& Schuerer, D. J. (2009). The impact of a simple, low-cost oral care protocol on ventilator-associated pneumonia rates in a surgical intensive care unit. J Intensive Care Med, 24(1), 54-62.

Tricco, A. C., Lillie, E., Zarin, W., O'Brien, K. K., Colquhoun, H., Levac, D., Moher, D., Peters, M. D. J., Horsley, T., Weeks, L., Hempel, S., Akl, E. A., Chang, C., McGowan, J., Stewart, L., Hartling, L., Aldcroft, A., Wilson, M. G., Garritty, C., Lewin, S., Godfrey, C. M., Macdonald, M. T., Langlois,

E. V., Soares-Weiser, K., Moriarty, J., Clifford, T., Tuncalp, O., \& Straus, S. E. (2018). PRISMA Extension for Scoping Reviews (PRISMA-ScR): Checklist and Explanation. Ann Intern Med, 169(7), 467-473.

Webster, J. (2016). A Odontologia Hospitalar a serviço do cidadão. CFO. https://website.cfo.org.br/a- odontologia-hospitalar-a-servico-do-cidadao/

Willis, P. J. (1965). The Role of Dentistry in the Hospital. J Am Dent Soc Anesthesiol, 12, 40-44.

Yokota, T., Tachibana, H., Konishi, T., Yurikusa, T., Hamauchi, S., Sakai, K., Nishikawa, M., Suzuki, M., Naganawa, Y., Hagihara, T., Tsumaki, H., Kubo, T., Sato, M., Taguri, M., Morita, S., Eguchi, T., Kubota, K., \& Zenda, S. (2016). Multicenter phase II study of an oral care program for patients with head and neck cancer receiving chemoradiotherapy. Support Care Cancer, 24(7), 3029-3036. 